\title{
El fracaso renal agudo por mieloma múltiple: cuidados de los pacientes en una unidad de hemodiálisis
}

\author{
Víctor Cantín Lahoz, Elisa Paula Rojo Zulaica, Ana Vecino Soler, Mercedes Marcén Letosa, Blanca Aznar Buil \\ Diplomados/as Universitaria de Enfermería. Unidad de Diálisis Hospital Clinico Universitario Lozano Blesa
}

\begin{abstract}
Resumen
El mieloma múltiple (MM) es una neoplasia hematológica con proliferación anormal de inmunoglobulinas y sus cadenas en sangre. La insuficiencia renal es una complicación grave y frecuente que puede derivar en fracaso renal en el $20 \%$ de los casos y puede requerir de terapia renal sustitutiva. Al debutar con una clínica tan inespecífica es importante un diagnóstico y tratamiento precoz para evitar el daño renal ya que agrava las complicaciones y condiciona la supervivencia. La depuración sanguínea mediante técnicas extracorpóreas con filtros de alto poro o high cut-off ( $\mathrm{HCO}$ ) son muy eficaces eliminando el $60-90 \%$ de las cadenas y recuperando la función renal en $50-80 \%$ de los casos. Debido a las características de estas sesiones prolongadas y la complejidad del tratamiento se requiere dedicación exclusiva por parte de enfermería durante todo el proceso.
\end{abstract}

Métodos: 9 pacientes diagnosticados de MM con cifras de más de $500 \mathrm{mg} / \mathrm{dl}$ de cadenas ligeras tratados con filtros $\mathrm{HCO}$ durante al menos 6 sesiones consecutivas de 6 horas de duración.

Resultados: Se realizaron 107 sesiones y se recuperó la función renal en 7. Complicaciones: 18 problemas de flujo, 12 episodios de coagulación del circuito, 13 fiebre, 14 hipotensiones, 13 transfusiones, 5 tratamientos con ansiolítico y 7 recambios de catéter.

Conclusiones: Las hemodiálisis de larga duración con filtros $\mathrm{HCO}$ son eficaces para eliminar las cadenas ligeras y recuperar la función renal. El papel de la enfermería

\begin{tabular}{|c|}
\hline Correspondencia: \\
Víctor Cantín Lahoz \\
Unidad de Diálisis Hospital Clínico U. \\
Lozano Blesa \\
C/. San Juan Bosco s/n \\
50009 Zaragoza \\
E-mail: victorcantin@gmail.com \\
\hline
\end{tabular}

es importante englobando una técnica compleja y dando una respuesta humana al debut de una enfermedad tan grave.

PALABRAS CLAVE:

- MIELOMA MÚLTIPLE

- FILTROS ALTO PORO

- FRACASO RENAL AGUDO

- CUIDADOS ENFERMERÍA

- HEMODIÁLISIS

Acure renal failure caused by multiple myeloma: Care in the hemodialysis unit

\begin{abstract}
Multiple myeloma ( $\mathrm{MM}$ ) is a hematologic malignancy with abnormal proliferation of immunoglobulins and its chains in blood. Renal insufficiency is a frequent and serious complication that can lead to a renal failure in $20 \%$ of cases and may require renal replacement therapy. As it starts with non-specific symptoms, it's important an early treatment to prevent renal damage in order to avoid complications and improve survival conditions. The extracorporeal blood purification techniques using high pore filters or high cut- off ( $\mathrm{HCO}$ ) are very effective because they remove $60-90 \%$ of the chains and recover renal function in $50-80 \%$ of the cases. Due to the long time sessions characteristics and the treatment complexity, nurses are required an exclusive dedication during the entire process.
\end{abstract}

Methods: 9 patients diagnosed of M M with figures over $500 \mathrm{mg} / \mathrm{dL}$ light chains treated with $\mathrm{HCO}$ filters for at least 6 consecutive sessions of 6 hours. 
Results: A total of 107 sessions were performed and renal function was recovered in 7 cases. Complications: 18 flow problems, 12 circuit coagulation episodes, 13 fever episodes, 14 hypotension, 13 transfusions, 5 anxiolytic treatment and 7 catheter changes.

Conclusions: The long term with haemodialysis $\mathrm{HCO}$ filters are effective in removing the light chains and recovering the renal function. The role of nursing is important including a complex technique and giving a human response to the debut of this severe disease.

\section{KEYWORDS:}

- MULTIPLE MYELOMA

- HIGH PORE FILTERS

- ACUTE RENAL FAILURE

- ACUTE KIDNEY INJURY

- NURSING CARE

- HAEMODIALYSIS

\section{Introducción}

El mieloma múltiple ( $\mathrm{MM}$ ) es una enfermedad neoplásica hematológica caracterizada por una proliferación anormal de células plasmáticas en la médula ósea ${ }^{1}$. Estas células provocan una liberación excesiva de inmunoglobulinas y sus cadenas (kappa o lambda) en sangre. Una de las complicaciones frecuentes y graves del M M es la insuficiencia renal, que aparece en la mitad de los casos por el efecto nocivo de las cadenas ligeras libres $(C L L)^{2}$. En condiciones normales se eliminan por el riñón, pero la producción en el MM supera con creces la capacidad de eliminación renal, con lo que se sobrecargan los receptores y se desbordan en el túbulo distal. Allí las CLL se unen a unas proteínas Ilamadas TammHorsfall, formando cilindros que obstruyen el flujo al precipitar dando lugar a una nefropatía por cilindros o el riñón de mieloma ${ }^{2-4}$. Aparece fracaso renal agudo (FRA) en el $20 \%$ de los casos, de los cuales la mitad de ellos requieren terapia renal sustitutiva. Esta situación se ve agravada por la deshidratación, hipercalcemia, fármacos nefrotóxicos, hiperuricemia y por la hiperviscosidad de la sangre ${ }^{2,3}$.

EI MM ocupa el $1 \%$ de las enfermedades neoplásicas y es el $13 \%$ de los cánceres hematológicos 5 . La edad media al diagnóstico es de 66 años y afecta más a varones $^{6}$. La esperanza de vida en el MM es inferior a un año si hay fracaso renal agudo y con las nuevas terapias puede ser de 5-7 años ${ }^{2,6,7}$.
La clínica es inespecífica hasta que aparecen los primeros síntomas como lesiones o dolores óseos, anemia, fallo renal, fatiga, hipercalcemia o disminución de peso ${ }^{2-6}$. Es muy importante el diagnóstico y tratamiento precoz de la enfermedad ya que el deterioro es potencialmente reversible con el tratamiento sintomático ${ }^{2,3}$. El problema más frecuente y condicionante es la insuficiencia renal que aparece en la mitad de los casos en algún punto de la enfermedad. Aumenta la carga tumoral, disminuye la calidad de vida, provoca mayor agresividad y aumenta las complicaciones y la mortalidad ${ }^{2-4}$.

Los nuevos fármacos quimioterápicos han revolucionado el tratamiento del $\mathrm{MM}$, pudiendo revertir el daño renal en muchos casos al frenar la producción de dichas CLL. Es el caso del bortezomib (inhibidor del proteaso$\mathrm{ma})$, que no requiere ajuste de dosis, asociado a dexametasona o de otros como la talidomida o lenalidomida (inmunomoduladores) ${ }^{2-4 .}$

Hay situaciones que requieren de un tratamiento coadyuvante depurativo de las CLL en sangre. Se determinan con un test específico que aporta información sobre la inclusión y continuidad del tratamiento depurativo ${ }^{8-9}$. Los métodos clásicos de depuración extracorpórea como la hemodiálisis o la plasmaféresis no permiten un aclaramiento eficaz de las CLL. En la diálisis convencional los filtros del dializador no tienen el tamaño de poro adecuado para eliminarlas y hay un aclaramiento mínimo. En la plasmaféresis sólo se elimina un $10-20 \%$ de las CLL que están en el espacio intravascular, mientras que el resto está en el espacio extravascular lo que dificulta su eliminación con los recambios de volumen plasmático, además de una pérdida importante de proteínas esenciales ${ }^{10-13}$.

Otro tipo de técnica es la hemodiafiltración con regeneración del ultrafiltrado mediante adsorción de resina (HFR supra) que utiliza convección, adsorción y difusión con un filtro dializador de doble cámara ${ }^{14,15}$. Aunque es algo más económico, son pocos los estudios realizados, en muy pocos pacientes y parece útil sólo en el tipo de cadenas kappa, no en las lambda.

Recientemente se han desarrollado otro tipo de membranas Ilamadas de alta permeabilidad, alto poro o high cut-off ( $\mathrm{HCO}$ ) que sí son eficaces para reducir las cadenas ligeras en sangre (hasta el 90\%) durante sesiones prolongadas de 5-8 horas ${ }^{16}$. Está demostrado que un diagnóstico y tratamiento precoz en la disminución de las CLL en las 3 primeras semanas, se asocia con un aumento significativo de la supervivencia ${ }^{17}$. El tamaño del poro es efectivo para las CLL, pero conlleva la pérdida 
de proteínas a través del dializador lo que requiere administrar albúmina al final de cada sesión.

Debido a la complejidad del tratamiento ya que precisa de minuciosos controles analíticos, monitorización de constantes, administración de fármacos, prevención de complicaciones y cuidados del acceso vascular, es necesario un control exhaustivo por parte de enfermería durante todo el proceso englobando una técnica compleja, así como dando la respuesta humana a un debut de una enfermedad que pone en compromiso la vida.

Presentamos la evolución de 9 pacientes que ingresaron en nuestra unidad de diálisis del Hospital Clínico Universitario Lozano Blesa de Zaragoza y se sometieron a este tratamiento.

\section{Pacientes y métodos}

Nueve pacientes entre Julio de 2011 y Noviembre de 2013 diagnosticados de nuevo MM y con FRA con cifras de CLL en suero mayor de $500 \mathrm{mg} / \mathrm{L}$. Debido a la aceptación del tratamiento por parte del hospital y de disponer de los medios técnicos y personales (nefrólogo, hematólogo y enfermería disponibles para hacer las guardias) fuimos el centro de referencia de Aragón. Para la realización del estudio se realizó una búsqueda de las historias clínicas de cada paciente, analizando las gráficas de enfermería de nuestra unidad y las unidades de hospitalización, además de la evolución clínica.

Para la realización de la técnica de depuración extracorpórea se emplearon monitores tipo Hospal Integra ${ }^{\circledR}$ equipados con ultrafiltros $1.4 \mathrm{~m} 2$ con tomas de agua ultra pura, ácido y bicarbonato proveniente de circuito cerrado. Los equipos eran de la marca Hospal ${ }^{\circledR}$ y el capilar empleado era la membrana de alto poro ( $\mathrm{HCO}$ ) Theralite ${ }^{\circledR}$ de $2.1 \mathrm{~m}^{2}$ de superficie y 45KD de tamaño de poro de la marca Gambro®.

La duración de las sesiones fue de 6 horas durante 6 días consecutivos. Si en estas sesiones no disminuían las cadenas ligeras por debajo de $500 \mathrm{mg} / \mathrm{L}$ se continuaba el tratamiento a días alternos hasta recuperar la función renal.

El cebado del circuito se realizó con el preparado comercial Prontoprime ${ }^{\circledR} 2000 \mathrm{ml}$ a una velocidad de 100 $\mathrm{ml} / \mathrm{h}$ salvo en el cebado de baño que se aumentaba a $200 \mathrm{ml} / \mathrm{h}$ con una ultrafiltración de $2 \mathrm{~kg} / \mathrm{h}$. Era importante un minucioso cebado del dializador para evitar en lo máximo posible la presencia de aire, que favoreciera su coagulación y disminuyera su eficacia dialítica.
El acceso vascular de los pacientes era un catéter temporal de localización yugular. Los flujos sanguíneos eran de 250-300 $\mathrm{ml} / \mathrm{min}$. El flujo de baño era de $500 \mathrm{ml} / \mathrm{min}$, conductividad de sodio a $140 \mathrm{mmol}$ y temperatura $37^{\circ} \mathrm{C}$. La pérdida de peso programada era mínima (entre 0.1 y $0.3 \mathrm{~kg} / \mathrm{h}$ ) reponiendo con suero fisiológico si era necesario. La heparina de inicio era un bolo de $2.5 \mathrm{ml}$ de heparina sódica al 1\% (2500 UI) y durante la diálisis en perfusión continua a $3 \mathrm{ml} / \mathrm{h}$ diluida en suero fisiológico (1500 UI) con un tiempo pre-paro de 30 minutos.

Durante la última hora de la sesión se administraba albúmina humana al $20 \% 100 \mathrm{ml}$. Además de gluconato cálcico, sulfato de magnesio, epoetina beta o concentrados de hematíes si precisaban tras el control analítico. El sellado de los catéteres se realizaba con heparina sódica al $1 \%$ con la cantidad marcada por el catéter.

Se realizaron analíticas de sangre midiendo las cadenas ligeras con el sistema Freelite the Binding Site ${ }^{\circledR}$ pre y post diálisis, además de otros parámetros como urea, creatinina, iones, magnesio, calcio iónico, hemograma y a mitad de la diálisis se medía la albúmina sérica. También se sacaron serologías y pruebas cruzadas al comienzo del tratamiento.

En la gráfica de enfermería se anotaban los flujos de sangre horarios, las presiones venosas, la ultrafiltración horaria, las tensiones arteriales, pulso, temperatura, administración de medicación, extracción de muestras y las incidencias y observaciones durante la diálisis.

El tratamiento quimioterápico estaba pautado por el servicio de hematología del hospital siendo en todos los casos ciclos de Bortezomib con Dexametasona y el tratamiento depurativo por el servicio de nefrología.

La introducción de esta nueva prestación en la cartera de servicios ha conllevado una redistribución de los recursos humanos y de los espacios en la unidad dejando a una enfermera con dedicación exclusiva y utilizando un cuarto para estos tratamientos.

\section{Resultados}

Los pacientes del estudio fueron 9 de los cuales 8 eran varones y 1 era mujer. La edad media fue de 60,33 años. (tabla 1). La procedencia fue exclusivamente de nuestra comunidad autónoma.

El tipo de cadena ligera fue inmunoglobulina lambda en 5 pacientes y Bence Jones kappa en los otros 4. Se realizaron biopsias en 5 de los pacientes con resultado de glomerulopatía por depósitos. 
Tabla I.

\begin{tabular}{|c|c|c|c|c|}
\hline Caso & Sexo & Edad & Tipo de cadena & Biopsia \\
\hline $\mathbf{1}$ & Varón & 57 & Lambda & $\mathrm{Si}$ \\
\hline $\mathbf{2}$ & Varón & 49 & Kappa & No \\
\hline $\mathbf{3}$ & Varón & 67 & Kappa & No \\
\hline $\mathbf{4}$ & Varón & 64 & Lambda & No \\
\hline $\mathbf{5}$ & Varón & 42 & Kappa & $\mathrm{Si}$ \\
\hline $\mathbf{6}$ & Mujer & 69 & Lambda & $\mathrm{No}$ \\
\hline $\mathbf{7}$ & Varón & 61 & Lambda & $\mathrm{Si}$ \\
\hline $\mathbf{8}$ & Varón & 63 & Lambda & $\mathrm{Si}$ \\
\hline $\mathbf{9}$ & Varón & 71 & Kappa & $\mathrm{Si}$ \\
\hline
\end{tabular}

Las cifras de cadenas ligeras antes del tratamiento fueron $11685 \mathrm{mg} / \mathrm{L}$ de media [4320-20200 mg/L] (tabla 2) y se redujeron hasta $145,14 \mathrm{mg} / \mathrm{L}$ de media $[30,3$ $577 \mathrm{mg} / \mathrm{L}]$.

El porcentaje de disminución de las CLL desde el principio del tratamiento al final fue del $98.62 \%$ de media.

La recuperación de la función renal al final del tratamiento se dio en 7 casos, mientras que 2 casos requirieron de terapia renal sustitutiva con hemodiálisis y diálisis peritoneal.

Dos pacientes fallecieron posteriormente al recaer del MM.

Se realizaron un total de 107 sesiones, de media 11,8 sesiones por paciente [6-27]. (tabla 3).

Todos los pacientes eran portadores de catéter temporal yugular. Se realizaron un total de 7 recambios de de catéter en 5 pacientes. Incluso en el paciente 1 se le puso un catéter permanente tipo Tesio por la duración de las sesiones (27).

Los flujos medios fueron de $250-300 \mathrm{ml} / \mathrm{min}$ como estaban pautados, pero en 4 pacientes y durante 18 sesiones se registraron problemas de flujo o de obstrucción con cifras menores de $200 \mathrm{ml} / \mathrm{min}$.

La coagulación del circuito, cámaras o del dializador teniendo que cambiarlo por otros nuevos se dieron en 6 pacientes durante 12 sesiones.

Los episodios de hipotensión con cifras de tensión arterial menores de $100 \mathrm{~mm} / \mathrm{Hg}$ o sensación manifiesta de bajada de tensión con sudoración, náuseas o malestar se dieron en 6 pacientes durante 14 sesiones resolviendo infundiendo suero.

Se objetivaron 13 episodios febriles en 6 pacientes con cifras superiores de $37.5^{\circ} \mathrm{C}$ y se administró tratamiento antibiótico en 4 pacientes durante 11 sesiones, los mismos pacientes que se realizó el cambio de catéter.

Se transfundieron a 7 pacientes durante 13 sesiones.

En 3 pacientes durante 5 sesiones se administró tratamiento ansiolítico con Lorazepam.

Detalladamente la serie de casos que fueron tratados en nuestro hospital son los siguientes:

\section{Caso 1:}

Varón de 57 años que ingresa en nuestro hospital el 13/05/2011. Tras confirmación por biopsia renal, se decide instaurar tratamiento hematológico y nefrológico conjunto.

Se le realizan un total de 27 sesiones de hemodiálisis con filtro $\mathrm{HCO}$ con las siguientes complicaciones: dos recambios de catéter (yugular y tesio) por mal funcionamiento en 12 episodios, 3 eventos de coagulación de

Tabla II.

\begin{tabular}{|c|c|c|c|c|c|}
\hline Caso & CLL antes $\mathrm{mg} / \mathrm{dl}$ & CLL después & $\begin{array}{c}\text { Porcentaje } \\
\text { disminución CLL }\end{array}$ & $\begin{array}{l}\text { Recuperación } \\
\text { función renal }\end{array}$ & Evolución \\
\hline 1 & 12900 & 577 & $95,52 \%$ & $\mathrm{Si}$ & Exitus \\
\hline 2 & 16210 & 93,8 & $99,42 \%$ & $\mathrm{Si}$ & Buena \\
\hline 3 & 7110 & 132 & $98 \%$ & $\mathrm{Si}$ & Exitus \\
\hline 4 & 17900 & 200 & $98,88 \%$ & $\mathrm{Si}$ & Buena \\
\hline 5 & 4320 & 44,7 & $98,96 \%$ & $\mathrm{Si}$ & Buena \\
\hline 6 & 10200 & 31,8 & $99,69 \%$ & $\mathrm{Si}$ & Buena \\
\hline 7 & 5830 & 132 & $97,73 \%$ & No & Diálisis \\
\hline 8 & 20200 & 64,7 & $99,67 \%$ & No & Diálisis \\
\hline 9 & 10500 & 30,3 & $99,7 \%$ & $\mathrm{Si}$ & Buena \\
\hline
\end{tabular}


Tabla III.

\begin{tabular}{|c|c|c|c|c|c|c|c|c|}
\hline Caso & $\begin{array}{l}\text { Número de } \\
\text { sesiones }\end{array}$ & $\begin{array}{l}\text { Cambio de } \\
\text { catéter }\end{array}$ & Mal funcionamiento & $\begin{array}{l}\text { Coagulación } \\
\text { líneas/dializador }\end{array}$ & Hipotensión & Fiebre & Transfusiones & $\begin{array}{c}\text { Tratamiento } \\
\text { ansiolítico }\end{array}$ \\
\hline 1 & 27 & 2 & 12 & 3 & 1 & 2 & 3 & No \\
\hline 2 & 7 & 1 & 2 & 1 & No & 1 & 1 & No \\
\hline 3 & 9 & No & No & No & No & 4 & 2 & 1 \\
\hline 4 & 7 & No & No & No & 2 & No & 3 & 3 \\
\hline 5 & 13 & 1 & 1 & 4 & No & 1 & No & 1 \\
\hline 6 & 6 & No & 1 & No & 5 & No & 1 & No \\
\hline 7 & 17 & 1 & 2 & 4 & 1 & 1 & 2 & No \\
\hline 8 & 9 & 2 & No & No & 4 & 4 & 1 & No \\
\hline 9 & 12 & No & No & No & 1 & No & No & No \\
\hline TOTAL & 107 & 7 & 18 & 12 & 14 & 13 & 13 & 5 \\
\hline
\end{tabular}

líneas y capilar por flujos bajos, 3 transfusiones, 1 episodio de hipotensión y 2 episodios de fiebre intradiálisis.

No se le realiza plan de cuidados en la unidad de hospitalización.

El 29/08/2011 se le da el alta hospitalaria por mejoría del proceso hematológico, habiendo recuperado la función renal.

El paciente sufre una recaída de su mieloma pasado un año con nueva afectación renal con resultado de muerte.

\section{Caso 2:}

Varón de 49 años que ingresa en nuestro hospital el 04/04/2010. No se realiza biopsia renal.

Se le realizan 7 sesiones de hemodiálisis con filtro $\mathrm{HCO}$ con las siguientes complicaciones: 1 recambio de catéter temporal yugular, 2 episodios de mal funcionamiento, 1 evento de coagulación de líneas, 1 episodio de fiebre y 1 transfusión.

El plan de cuidados en la unidad de hospitalización consta sólo de intervenciones de proceso: cuidados de catéter vascular central, periférico y urinario, y como complicaciones potenciales dolor e hipertermia.

El paciente es dado de alta por mejoría el 20/04/2012, no requiriendo diálisis por recuperación total de la función renal.

\section{Caso 3:}

Varón de 67 años que ingresa el 29/03/2012. No se realiza biopsia renal.

Se le realizan 9 sesiones de hemodiálisis con filtro $\mathrm{HCO}$ sin que quede reflejado complicaciones de la técnica.
Requiere de 1 tratamiento ansiolítico en el trascurso de las sesiones además de 4 episodios febriles y 2 transfusiones.

Es valorado en la unidad de hospitalización con conocimientos deficientes y se registran como complicaciones potenciales dolor, hemorragia, hipertermia, taquiarritmia y riesgo de infección.

Es dado de alta con fecha de 24/04/2012 por mejoría recuperando su función renal. El paciente fallece al poco tiempo por un shock séptico.

\section{Caso 4:}

Varón de 67 años que ingresa con fecha de 28/05/2012. No se realiza biopsia renal.

Se realizan un total de 7 sesiones de hemodiálisis con filtro HCO. Como incidencias sufre de 2 episodios de hipotensión. El paciente requiere tratamiento ansiolítico en 3 sesiones y en las sesiones sufre varios episodios de diarreas líquidas y melenas. La función renal se recupera.

Es valorado en la unidad de hospitalización con conocimientos deficientes, y como complicaciones potenciales tiene diarrea, hemorragia, hipertermia, trastornos del sueño y ansiedad/angustia adaptativa. La evolución es mala con exitus el 08/07/2012.

\section{Caso 5:}

Varón de 42 años que ingresa el 06/06/2012. Sí se le realiza biopsia renal.

Se realizan un total de 13 sesiones de hemodiálisis con filtro HCO. Como complicaciones sufrió coagulación de circuito en 4 sesiones distintas, un episodio de malfun- 
cionamiento y precisó de un recambio de catéter temporal yugular. Cursó con 1 episodio de ansiedad, 1 de hipertermia y se le transfundió en 1 ocasión.

En la unidad de hospitalización es valorado con los siguientes diagnósticos enfermeros: conocimientos deficientes y déficit de autocuidado (alimentación). Como complicaciones potenciales se registra dolor, ansiedad, hipertermia, trastornos del sueño y angustia adaptativa.

Se recupera del fallo renal y es dado de alta el 03/08/12 con buena evolución.

\section{Caso 6:}

Mujer de 69 años que ingresa en nuestro hospital con fecha de 20/07/12. No se le realiza biopsia renal.

Se realizan un total de 6 sesiones de hemodiálisis con filtro HCO. Durante éstas no sufre ningún tipo de complicación como coagulación o recambio de catéter, sólo 1 problema de malfuncionamiento. Sin embargo cursa recurrentes episodios de hipotensión (en 4 de las 6 sesiones) llegando a perder la conciencia en una de ellas. Se transfunde en 1 sesión.

Es valorada en la unidad de hospitalización con diagnóstico enfermero de estreñimiento, y como complicaciones potenciales se registran dolor e insuficiencia renal.

Finalmente recupera fallo renal y es dada de alta el 10/08/12 con buena evolución.

\section{Caso 7:}

Varón de 61 años que ingresa en nuestro hospital con fecha de 24/02/13.

Se realizan un total de 17 sesiones de diálisis con filtro $\mathrm{HCO}$, Se realiza un recambio de catéter temporal yugular y aparecen las siguientes complicaciones: 4 episodios de coagulación de circuito, 2 problemas de malfuncionamiento, 1 episodio de hipotensión, 1 de fiebre y 2 transfusiones.

En la unidad de hospitalización es valorado con el diagnóstico enfermero de conocimientos deficientes. Como complicaciones potenciales se señalan diarrea e hipertermia.

No recupera fallo renal, continúa tratamiento sustitutivo renal con hemodiálisis convencional (durante el cual requiere de un cambio de catéter temporal) y posteriormente continúa tratamiento renal sustitutivo en diálisis peritoneal. Es dado de alta con fecha de 08/04/13.

\section{Caso 8:}

Varón de 63 años ingresa en nuestro hospital con fecha de $27 / 02 / 13$.
Se realizan un total de 9 sesiones de hemodiálisis con filtro $\mathrm{HCO}$, durante las cuales precisa de dos recambios de catéter temporal yugular, aunque no aparecen problemas de flujo o malfuncionamiento. Cursa recurrentes episodios de hipotensión (en 4 de 9 sesiones), además de 4 episodios de hipertermia y requiere de 1 transfusión.

En la unidad de hospitalización es valorado con el diagnóstico enfermero de conocimientos deficientes, déficit de autocuidado (vestido), y como intervenciones de proceso se registra el mantenimiento del catéter de diálisis. Como complicaciones potenciales se señalan diarrea e hipertermia.

No recupera fallo renal, continúa tratamiento sustitutivo renal con hemodiálisis y posteriormente se le realiza FAVI. Tiempo después ésta deja de funcionar y se le coloca catéter fijo tunelizado tipo Tesio. Es dado de alta el 13/04/13.

\section{Caso 9:}

Varón de 71 años que ingresa en nuestro hospital con fecha de 04/11/13.

Se realizan un total de 12 sesiones de hemodiálisis con filtro $\mathrm{HCO}$, durante las cuales no precisa de recambio de catéter, ni episodios de coagulación. Únicamente cursa con 1 episodio de hipotensión a lo largo de las sesiones.

En la unidad de hospitalización es valorado con el diagnóstico enfermero de conocimientos deficientes, y como intervenciones de proceso se registra el mantenimiento del catéter de diálisis. Como complicaciones potenciales se señalan diarrea e hipertermia.

Se recupera del fallo renal y es dado de alta el 05/12/13 con buena evolución.

\section{Discusión}

La recuperación y supervivencia del MM está muy ligada al grado de afectación renal y puede variar de varios meses a poco más de un año $0^{2,3}$. Es importante un diagnóstico precoz, pero se confirma la dificultad del diagnóstico como queda reflejado en otros artículos debido a la variabilidad de signos y síntomas en su debut como anemia, fallo renal o fracturas óseas ${ }^{2-6}$. Una analítica de sangre 0 una radiografía puede ser el inicio para el diagnóstico del MM, además del test específico de cadenas ligeras en sangre ante sospecha. Una vez diagnosticado es importante instaurar el tratamiento quimioterápico sobre todo si hay afectación renal y evitar un deterioro mayor que dificulte la recuperación y provoque una dis- 
minución en la calidad de vida y la supervivencia ${ }^{4}$. En caso de IRA se hace necesario además una monitorización de la función renal, de las CLL y depuración extracorpórea de las mismas ${ }^{9,15}$.

Los primeros tratamientos que aparecieron eliminaban las cadenas por plasmaféresis, con escasos resultados de recuperación funcional (mejoría del filtrado glomerular, dependencia de diálisis o reducción de la mortalidad $)^{10}$. Los estudios son de series cortas de pacientes ${ }^{12,13}$ precisan de tratamientos prolongados de dos meses y no se reduce de manera significativa las cadenas ligeras en esas sesiones ${ }^{10-13}$.

Recientemente han aparecido los filtros HFR supra ${ }^{14}$ que disminuye en sangre las cadenas en un $38-63 \%{ }^{15}$, pero parece que es más efectivo sólo en las cadenas tipo kappa (22kD) y no en las lambda (45kD) por el tamaño del poro de estos filtros $(42 k D)$. Se utiliza un dializador de doble cámara, una de ellas de alto flujo y otra de baja permeabilidad, ultrafiltrando en la primera, haciendo adsorción con una cámara de resina y reinfundiendo antes la segunda cámara. Son pocos pacientes los analizados y se basan principalmente en el ahorro económico del tratamiento al no perder proteínas ${ }^{15}$.

El tratamiento con mejores resultados hasta el momento son los filtros de alto poro descritos por Hutchison ${ }^{16}$ y que permiten reducir el $60-90 \%{ }^{16-17,19-23}$ de las cadenas ligeras libres en sangre. Además recupera la función renal en $50-80 \%{ }^{16-17,19-23}$ de los casos aumentando la supervivencia de 8 a 42 meses. El tamaño del poro permite el paso de las cadenas ligeras, pero hay una pérdida de proteínas en el dializador y hay que reponer con albúmina. Estos filtros no llevan mucho en el mercado y al principio se utilizaba el dializador HCO 1100 individual 0 en paralelo ${ }^{19-20}$, de $1.1 \mathrm{~m}^{2}$ y actualmente el de 2100 Theralite $\circledast$ de $2.1 \mathrm{~m}^{2}$ por lo que los estudios son muy variados, con muestras pequeñas, en muchos casos sólo son casos clínicos de uno o dos ${ }^{9,24}$ y con diferente metodología ${ }^{19,25,26}$.

En nuestro estudio recuperaron la función renal 7 de los 9 pacientes con cifras similares a otros estudios y las cifras de cadenas ligeras se redujeron porcentualmente. Los estudios encontrados se centran en valorar el porcentaje de reducción de cadenas y la efectividad del tratamiento, pero no registran apenas las complicaciones del mismo. En nuestras sesiones la complicación más frecuente fueron los problemas de flujo en 18 sesiones. En varios artículos no aparece ningún tipo de complicación al realizar este tratamiento, porque no se registraban en el estudio ${ }^{18-19,27}$ o porque no aparecieron 22,28-30. En el artículo de Martín Reyes ${ }^{20}$ con 6 pacien- tes y 60 sesiones el problema más frecuente descrito fue la coagulación del sistema que ocupó la mitad de las complicaciones, seguido de mal funcionamiento. En el estudio de Muñoz ${ }^{31}$ aparece 2 episodios de coagulación en 9 pacientes y el Mallol ${ }^{32}$ con 1 paciente casi todas las sesiones se coaguló el circuito de diálisis.

No se refleja la planificación de los cuidados en un proceso en el que el paciente pasa de un diagnóstico de una enfermedad grave como es el mieloma múltiple con mal pronóstico, afectación renal e inicio de un tratamiento nuevo para el. Esto se ve reflejado en la toma de ansiolíticos y la demanda continua de información. El papel de la enfermería vigilando y dando un apoyo constante en esta crisis de vida es muy importante.

Los registros enfermeros que manejamos en la unidad de hemodiálisis convencional resultan insuficientes para recoger correctamente estas sesiones de larga duración y complejidad de cuidados. Por lo cual hemos elaborado una nueva gráfica que permite plasmar la correcta realización de la técnica así como los cambios y la adaptación del paciente a la nueva situación. Esto nos permite disponer de los datos necesarios para planificar unos cuidados individualizados a cada situación.

Hay en marcha dos grandes estudios multicéntricos a nivel europeo que son el Eulite ${ }^{33}$ y el Myre ${ }^{34}$. El Eulite analizan en 90 pacientes de nuevo diagnóstico de MM se aleatorizarán con tratamiento de filtros de alto poro y filtros de alto flujo con un seguimiento de 24 meses analizando la supervivencia e independencia de hemodiálisis, siguiendo el mismo tratamiento quimioterápico. El Myre que busca utilizar bortezomib con dexametasona sólo o asociado a ciclofosfamida, además de tratar con filtros de $\mathrm{HCO}$ o con dializadores de alto flujo.

A pesar de todo serían necesarios actualizar los costes globales del tratamiento y valorar la calidad de vida en el MM. Sólo hay un artículo de Grima ${ }^{35}$ en el que mide el coste efectividad del tratamiento del MM utilizando los filtros $\mathrm{HCO}$ y hemodiálisis estándar. Se demuestra que aumenta la esperanza de vida de 20 meses a 34 meses utilizando los filtros $\mathrm{HCO}$, además de un ahorro de casi $6500 £(8025 €)$ en el tratamiento.

\section{Conclusión}

Queda de manifiesto la importancia del diagnóstico y tratamiento precoz en la enfermedad del mieloma múltiple. El método de depuración con filtros de alto poro es un método seguro y fiable. Es importante unificar los criterios de inclusión en este tipo de tratamiento, 
además hacer protocolos de actuación y recoger correctamente toda la información posible para seguir investigando en esta novedosa técnica. El papel de la enfermería durante el acompañamiento de los pacientes requiere de habilidades de cuidado propias de la profesión, además de un control óptimo de las técnicas del tratamiento y su seguimiento.

\section{Agradecimientos}

A toda la unidad de enfermería y el equipo médico de la Unidad de Hemodiálisis del Hospital Clínico Universitario Lozano Blesa.

\section{Bibliografía}

1. Kyle RA, Rajkumar SV. Multiple myeloma. N Eng J Med 2004;351:1860-73.

2. Palumbo A, Anderson K. Multiple myeloma. N Eng J Med 2011;364:1046-60.

3. Gaballa M, Laubach J, Schlossman R, Redman K, Noonan K, Mitsiades $S$ et al. Management of myeloma-associated renal dysfunction in the era of novel therapies. Expert Rec Hematol 2012;5(1):51-68.

4. Davenport A, Merlini G. Myeloma kidney: advances in molecular mechanisms of acute kidney injury open novel therapeutic opportunities. Nephrol Dial Transplant 2012;27:3713-8.

5. Raab MS, Podar K, Breitkreutz I, Richardson $P G$, Anderson KC. Multiple myeloma. Lancet 2009:374(9686):324-339.

6. Kyle RA, Gertz MA, Witzig TE, Lust JA, Lacy MQ, Dispenzieri A, et al. Review of 1027 patients with newly diagnoses multiple myeloma. Mayo Clin Proc 2003;78:21-33.

7. Martín Reyes G, Valera A, Frutos MA, Ramos B, Ordóñez V, López de Novales E. Supervivencia de pacientes con mieloma tratados con diálisis. Nefrología 2003;XXIII(2):131-136.

8. Hutchison $C A$, Plant $T$, Drayson $M$, Cockwell $P$, Kountouri M, Basnayake $K$ et al. Serum free light chain measurement aids the diagnosis of myeloma in patients with severe renal failure. BMC Nephrology 2008;9(11)
9. Martin MA, García SA, Caba M, Palacios ME, Gomez M, Claver C. Riñón de mieloma: importancia de la valoración de respuesta mediante monitorización de las cadenas ligeras libres en suero. Nefrología 2013;33(6):862-4.

10. Clark WF, Stewart AK, Roca GA, Sternbach M, DM Sutton, Barrett BJ et al. Plasma exchange when myeloma presents as acute renal failure. Ann Intern Med 2005;143:773-780.

11. Leung N, Gertz MA, Zeldenrust SR, Rajkumar SV, Dispenzieri A, Fervenza FC et al. Improvement of cast nephropathy with plasma exchange depends on the diagnosis and reduction of serum free light chains. Kidney Int 2008;73:1282-1288.

12. Levy F, Camarero V, Torres G, Hijazi B. Plasmaféresis como tratamiento adyuvante en el fallo renal agudo secundario al riñón de mieloma. Nefrología 2009;29(3):283-284.

13. Burnette BL, Leung N, Rajkumar SV. Renal improvement in myeloma with bortezomib plus plasma exchange. N Engl J Med 2011;364:23656.

14. Testa A, Dejoie T, Lecarrer $D$, Wratten $M$, Sereni $L$, Renaux JL. Reduction of free inmunoglobulin Light chains using adsorption properties of hemodiafiltration with endogenous reinfusion. Blood Purif 2010;30:34-6.

15. Pendón Ruiz MV, Alvarez MA, Ojeda R, Martín A, Cariacedo J, Caballero $\mathrm{J}$ et al. Eficacia de la hemodiafiltración con regeneración del ultrafiltrado en la reducción de las cadenas ligeras en el mieloma múltiple con insuficiencia renal. Nefrología 2013;33(6):788-96.

16. Hutchison CA, Bradwell AR, Cook M, Basnayake K, Basu S, Harding S et al. Treatment of acute renal failure secondary to multiple myeloma with chemotherapy and extended high cut-off haemodialysis. Clin J Am Soc Nephrol 2009;4:745754.

17. Hutchison CA, Cockwell $P$, Stringer $S$, Bradwell $A$, Cook M, Gertz MA, et al. Early reduction of serum-free light chains associates with renal recovery in myeloma multiple. J Am Soc Nephrol 2011;22:1129-36.

18. Álvarez MA, Martín A, Aljama P. Nuevas alternativas en el tratamiento del riñón del riñón de mieloma. Nefrología 2013;33(4):443-7. 
19. Kleeberg L, Morgera $S$, Jakob C, Hocher $B$, Schneider M, Peters $H$ et al. Novel renal replacement strategies for the elimination of serum free light chains in patients with kappa light chain nephropathy. Eur J Med Res. 2009;14(2)47-54.

20. Khalafallah A, Wuong $S$, Amor S, Mohamed M, Mace $\mathrm{R}$, Khalil R et al. Early application of high cutt-off haemodialysis for de-novo myeloma nephropathy is associated with long-term dialysis-independency and renal recovery. Mediterr Hematol J Infect Dis. 2013;5(1):

21. Martin Reyes G, Toledo R, Torres A, Sola E, Blanca $L$, Fuentes $L$ et al. Tratamiento con hemodiálisis del fracaso renal agudo en el mieloma múltiple con filtros de alto poro (high cut-off). Nefrol 2012;32(1):35-43.

22. Borrego J, Perez MP, Biechy M, Merino $E$, Sánchez MC, García MJ et al. Tratamiento con hemodiálisis larga con filtros de alto cut-off en la nefropatía por cilindros del mieloma: nuestra experiencia. Nefrología 2013;33(4):515-523.

23. Heyne N, Denecke B, Guthoff M, Oehrlein K, Kanz L. Extracorporeal light chain elimination: high cutoff ( $\mathrm{HCO}$ ) haemodialysis parallel to chemotherapy allows for a high proportion of renal recovery in multiple myeloma patients with dialysis-dependent acute kidney injury. Ann Hematol 2012;91:729735.

24. Shum HP, Chan KC, Chow CC, Kho B, Yan WW. Cast nephropathy with acute renal failure treated with high cut-off haemodialysis in a patient with multiple myeloma. Hong Kong Med J 2010;16(6):489-492.

25. Peters NO, Laurains E, Cridlig J, Hulin C, Cao-huu T, Frimat L. Impact of free light chain haemodialysis in myeloma cast nephropathy: a case-control study. Haemodialysis International 2011;15:538-545.

26. Hutchison CA, Heyne $N$, Airia $P$, Schindler $R$, Zickler $\mathrm{D}$, Cook $\mathrm{M}$ et al. Immunoglobulin free light chain levels and recovery from myeloma kidney on treatment with chemotherapy and high cut-off haemodialysis. Nephrol Dial Transplant 2012;27:3823-8.

27. Álvarez R, Martín F, Abascal JA, Berni A, Dourdil V, Palomera L. Tratamiento de la IRA secundaria a mieloma múltiple con filtro de high cut-off. Dial Traspl. 2013;34(1):36-40.
28. Sinisalo $M$, Silvennoinen $R$, Wirta 0 . High cut-off haemodialysis and bortezomib-based therapy to rescue kidneys in myeloma-dependent cast nephropathy. Am J of Hematology 2012; 87(6):640.

29. Li Cavoli G, Schillaci O, Zagarrigo C, Tralongo A, Servillo F, Passanante $S$ et al. Coment on "Haemodialysis using high cut-off dialysers for treating acute renal failure in multiple myeloma". Nefrología 2012;32(3):396.

30. Echabe L, Iturralde I, González E, Telleria A, Rodrigo T, Rivas M. Hemodiálisis con filtro de alta permeabilidad para eliminación de cadenas ligeras en pacientes con mieloma. Disponible en: http://www.revistaseden.org/files/2736_23\%20 \%201620.pdf con acceso 24 abril 2013.

31. Muñoz RI, Gallardo I, Neyra P, Torres A, Romero $M A$, Hernando $A$ et al. Hemodiálisis con membrana de alto poro en el fracaso renal agudo por cadenas ligeras del mieloma múltiple: resultados a 3 años de un estudio protocolizado en un centro. Póster presentado en el XLII congreso de la SEN el 7 de Octubre de 2012 en Gran Canaria.

32. Mallol A, Folch MJ, Renau E, Cerrillo V. Hemodiálisis con dializadores de poro grueso para tratar el fracaso renal agudo del paciente con mieloma. Rev Soc Esp Enferm Nefrol 2011;14(2):

33. Hutchison CA, Cook M, Heyne N, Wiesel $K$, Billingham L, Bradwell $A$ et al. European trial of free light chain removal by extended haemodialysis in cast nephropathy (EuLITE): A randomized control trial. Trials 2008;9:55.

34. Studies in patient with multiple myeloma and renal failure due to myeloma cast nephropathy (MYRE)

35. Grima DT, Airia P, Attard C, Hutchison CA. Modelled cost-effectiveness of high cut-off haemodialysis compared to standard haemodialysis in the management of myeloma kidney. CMRO 2011;27(2):383-391. 